\title{
Late Presentation of Pericarditis After Honey Bee Sting: Case report
}

\author{
Celaleddin Soyalp ${ }^{1}$, Suleyman Cagan Efe ${ }^{2}$
}

\section{Abstract}

Honey bees are members of the hymenopteran family. The sting of honey bees venom can cause a range of effects from irritation to severe form of anaphylactic shock. Anaphylactic shock occurring after honey bee sting is reported in literature for about $5 \%$ of cases. Pericarditis occurring due to honey bee sting is a rare complication, which was previously not reported. We reported a case of pericarditis following a honeybee sting with late clinical manifestations. In our case, ECG demonstrated pericarditis at the third day of hospitalization as a late complication

Key words: Anaphylactic shock, honey bee sting, pericarditis, late presentation

\section{Introduction}

Honey bee sting complications can range from abdominal pain, vomiting-nausea, headache, anxiety, itching, palpitations, and high blood pressure to severe form of anaphylactic shock. Anaphylactic shock occurring after honey bee sting is reported in literature for about 4.4-6.4 \% of cases (1,2). Pericarditis occurring due to honey bee sting is a rare complication, which was previously not reported. Kounis syndrome, myocardial infarction and myocarditis induced by allergic hypersensitivity and anaphylactoid reactions, was described in the literature. We reported a case of pericarditis following a honeybee sting with late clinical manifestations

\section{Case Presentation}

A 48-year-old woman was admitted to our hospital thirty minutes later after being stung. Her past medical history included only hypertension. The patient exhibited a mild drowsy mental state with urticarial rash in the neck and upper abdominal region. In the emergency room, periorbital and perioral edema subsequently developed. She became agitated, tachypneic with stridor, desaturated $(\mathrm{SaO} 2: 72 \%$ on oxygen mask) and hypotensive (Blood pressure was $80 / 50 \mathrm{mmHg}$ ) with moist extremities. After intravenous adrenaline $(2 \mathrm{mg})$, intravenous steroids (Hydrocortisone 100mg), antihistamines (Chlorpheniramine 10mg) and intravenous fluids were immediately given; she was intubated and subsequently transferred to the intensive care unit.
Her vital signs became more stable after an hour (blood pressure, 120/75 $\mathrm{mmHg}$; heart rate, $98 \mathrm{bpm}$; SpO2: 98\%). At the first day of hospitalization, electrocardiography (ECG) and high sensitivity troponin assays did not demonstrate any evidence of myocardial injury. On second day the patient was extubated.

Third day of hospitalization she complained of pleuritic substernal chest heaviness, and dyspnea. The laboratory results were as follows: leukocytes 14000 (normal range: 4-10×103), CRP (C-reaktif protein): $32 \mathrm{mg} / \mathrm{L}$ (normal value: $0-8)$, CK (Creatine Kinase): 140 (normal value: 55-170 u/L), CK-MB: 3 (< $5 \mathrm{ng} / \mathrm{ml})$ with mild elevation in the troponin I: $0.024(<$ $0.014 \mathrm{ng} / \mathrm{ml}$ ).

The other laboratory results were normal. ECG (Electrocardiography) showed significant ST-segment elevation in all leads except avr compatible with pericarditis (Figure 1). ECG showed normal left ventricular systolic and diastolic functions with no pericardial effusion.

At the second day of colchicine and ibuprofen treatment her complaints significantly decreased. The cardiac enzymes were returned to the normal values and the control echocardiography showed normal findings on the sixth day. The patient was discharged with ibuprofen and colchicine treatment. 


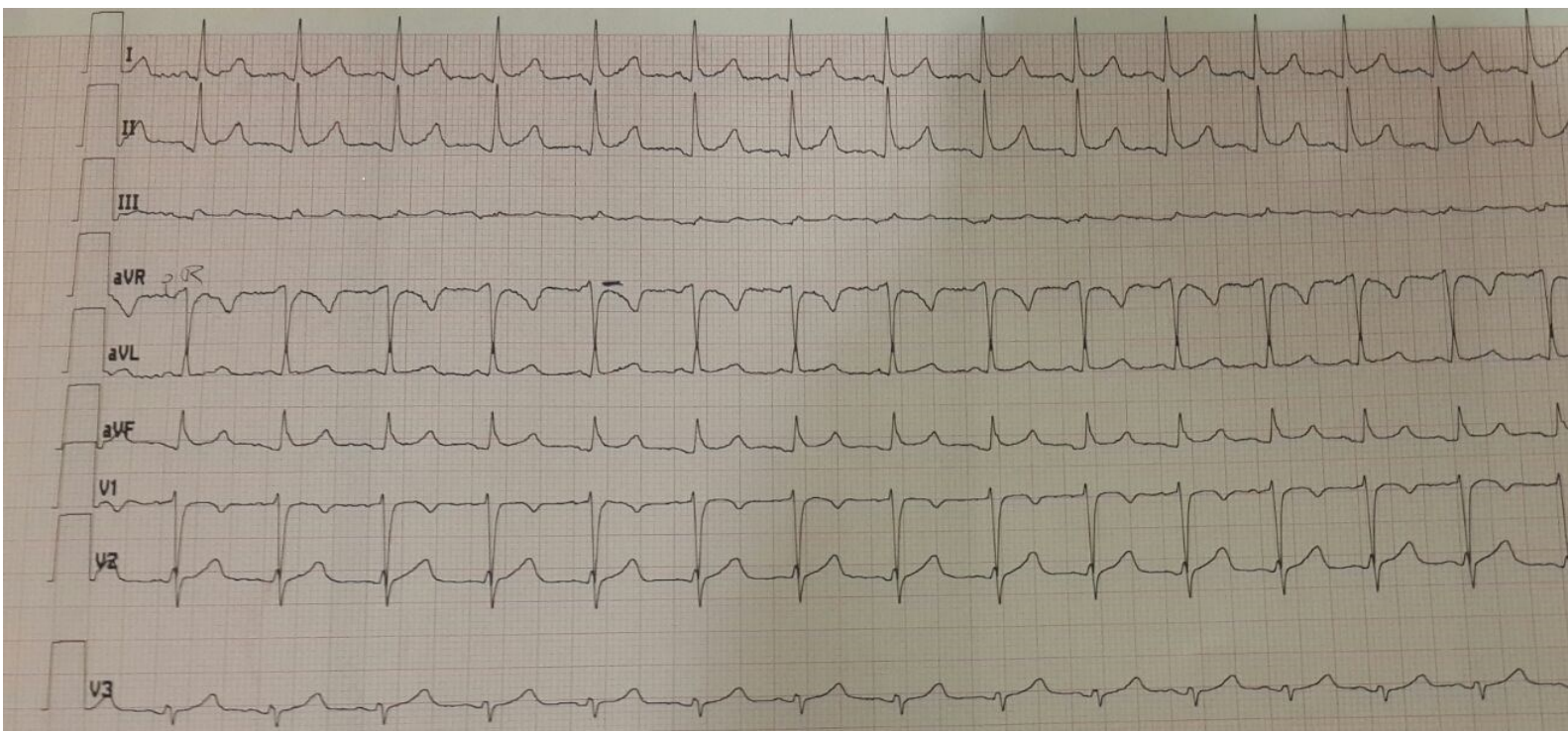

Figure 1: Electrocardiography of patient

\section{Discussion}

Honey bees are members of the hymenopteran family. The honey bee sting can cause a range of side effects from irritation to severe form of anaphylactic shock (3). Multi organ failures, rhabdomyolysis, electrocardiographic changes, acute kidney injury and myocardial infarction are well known complications $(4,5)$. Several cases of myocarditis and Kounis syndrome occurring after the honey bee sting have been reported. Type I Kounis syndrome includes patients with normal coronary arteries caused by acute release of inflammatory mediators during the allergic reaction (6). Epinephrine, dopamine and thromboxanes in honey bee venom can cause severe platelet aggregation, direct vasoconstriction and myocardial damage or dysfunction (7). Hypersensitivity myocarditis is diagnosed in the presence of eosinophils, atypical lymphocytes, and giant cells in myocardial biopsy, whereas biopsy in Kounis syndrome is typically normal (6). Late gadolinium enhancement in magnetic resonance imagination is highly specific for diagnosing of Kounis syndrome and myocarditis (8).

In our case, ECG demonstrated pericarditis at the third day of hospitalization as a late complication. Direct toxin effect on pericardial damage and inflammatory response secondary to allergenic effect of toxin may have an additional role in pericarditis mechanism.

Conflict of Interest: The authors declare no potential conflicts of interest with respect to the research, authorship, and/or publication of this article.

\section{References}

1. Witharana EW, Wijesinghe SK, Pradeepa KS, Karunaratne WA, Jayasinghe S. Bee and wasp stings in Deniyaya; a series of 322 cases. Ceylon Med J. 2015; 60(1):5-9.

2. Wijerathna BT, Rathnayake GK, Agampodi SK. Hornet stings presenting to a primary care hospital in Anuradhapura district in Sri Lanka. J Wildness \& Environmental Medicine 2014; 25: 122-6.

3. Gruchalla RS. Immunotherapy in allergy to insect stings in children. N Engl J Med 2004; 351: 707-9.

4. Kularatne SAM, Gawarammana IB, De Silva PHJG. Severe multiorgan dysfunction following multiple wasp (Vespa affinis) stings. Ceylon Med J 2003; 48: 146-7.

5. Levine HD. Acute myocardial infarction following wasp sting. Report of two cases and critical survey of the literature. Am Heart J 1976; 91:365-74.

6. Kounis NG, Mazarakis A, Tsigkas G, Giannopoulos S, Goudevenos J. "Kounis syndrome: a new twist on an old disease," Future Cardiology 2011; 7 (6):805-824.

7. Brasher GW, Sanchez SA. Reversible electrocardiographic changes associated with wasp sting anaphylaxis. JAMA 1974;229:1210-1211

8. Almpanis GC, A. Mazarakis DA. Dimopoulos et al. "The conundrumof hypersensitivity cardiac disease: hypersensitivity myocarditis, acute hypersensitivity coronary syndrome (Kounis syndrome) or both?" International Journal of Cardiology; 2011; 148(2):237-240.

Copyright ( 12014 The Author(s); This is an open-access article distributed under the terms of the Creative Commons Attribution License (http://creativecommons.org/licenses/by/4.0), which permits unrestricted use, distribution, and reproduction in any medium, provided the original work is properly cited. All Rights reserved by international journal of Medical Science and Discovery. 